This manuscript is a preprint and has been submitted to the Journal of the Geological Society. This manuscript has not undergone peer review. Subsequent versions of this manuscript may have different content. If accepted, the final peer-reviewed version of this manuscript will be available via the "Peerreviewed Publication' DOI link on the right-hand side of this webpage. Please feel free to contact any of the authors directly to comment on the manuscript. 


\title{
Reproducibility in subsurface geoscience
}

\author{
Michael J. Steventon ${ }^{1 *}$, Christopher A-L. Jackson², Matt Hall ${ }^{3}$, Mark T. Ireland ${ }^{4}$, Marcus Munafo ${ }^{5}$, \\ \& Kathryn J. Roberts ${ }^{6}$
}

Contact:m.steventon@shell.com

\author{
${ }^{1}$ Shell Research Ltd, Shell Centre, London, SE1 7NA, UK \\ ${ }^{2}$ Department of Earth and Environmental Sciences, The University of Manchester, Williamson Building, Oxford Road, \\ Manchester, M13 9PL, UK \\ ${ }^{3}$ Agile Scientific, PO Box 336, Mahone Bay, NS BOJ 2E0, Canada \\ ${ }^{4}$ School of Natural and Environmental Sciences, Newcastle University, Newcastle upon Tyne, NE1 7RU, UK \\ ${ }^{5}$ School of Psychological Science, Bristol Population Health Science Institute, University of Bristol, BS8 1TU, UK \\ ${ }^{6}$ Institute for Global Health, University College London, London, NW3 2PF, UK
}

\section{Abstract}

Reproducibility, the extent to which consistent results are obtained when an experiment or study is repeated, sits at the foundation of science. The aim of this process is to produce robust findings and knowledge, with reproducibility being the screening tool to benchmark how well we are implementing the scientific method. However, the re-examination of results from many disciplines has caused significant concern as to the reproducibility of published findings. This concern is wellfounded - our ability to independently reproduce results build trust both within the scientific community, between scientists and the politicians charged with translating research findings into public policy, and the general public. Within geoscience, discussions and practical frameworks for reproducibility are in their infancy, particularly in subsurface geoscience, an area where there are commonly significant uncertainties related to data (e.g. geographical coverage). Given the vital role of subsurface geoscience as part of sustainable development pathways and in achieving Net Zero, such as for carbon capture storage, mining, and natural hazard assessment, there is likely to be an increased scrutiny on the reproducibility of geoscience results. We surveyed 347 Earth scientists from a broad section of academia, government, and industry to understand their experience and knowledge of reproducibility in the subsurface. More than $85 \%$ of respondents recognised there is a reproducibility problem in subsurface geoscience, with $>90 \%$ of respondents viewing conceptual biases as having a major impact on the robustness of their findings and overall quality of their work. Access to data, undocumented methodologies, and confidentiality issues (e.g. use of proprietary data and methods) were identified as major barriers to reproducing published results. Overall, the survey results suggest a need for funding bodies, data providers, research groups, and publishers to build a framework and set of minimum standards for increasing the reproducibility of, and political and public trust in, the results of subsurface studies. 


\section{Introduction}

Definitions of reproducibility can vary between and within disciplines. Here, we broadly define reproducibility as the ability to confirm the results and conclusions of your own or others' work. The principle can be split further into (i) repeatability, where results are obtained under the same conditions by the same research team, (ii) replicability, where results are obtained by a different research team using the same methodology, and (iii) reproducibility, where results are obtained by a different research team using a different methodology and/or dataset. In addition to these, transparency, the requirement for access to data, software, documentation of methods, or metadata, for example, is a prerequisite of any study to facilitate repeatability, replicability and reproducibility. Concern over the reproducibility of scientific results has gained significant traction in recent years as large-scale reviews in disciplines such as medicine (Nosek et al. 2017), psychology (Open Science Collaboration, 2015), and economics (Camerer et al. 2016), have often cast doubt on the reliability of publish results. A recent survey of scientists from across various fields found that $>70 \%$ could not reproduce other scientists' experiments, and that $>50 \%$ had failed to reproduce their own work (Baker 2016). This challenge in reproducibility has been attributed to several factors including poor method descriptions, selective reporting, poor selfreplication, pressure to publish, and poor analyses/statistical power.

Some areas of Earth Science have been proactive in discussing and improving upon reproducibility, for example the reconstruction of palaeo-climate through evaluation of atmospheric and sea-level changes (e.g., Milne et al. 2009). This field has been heavily scrutinised because of its direct impact on human lives, economies, and public policy when predicting and understanding climate change. Subsurface geoscience has for the most part flown under the radar of global scrutiny, however, in the future it is likely areas in the subsurface such as carbon capture storage (CCS), mining, and natural hazard assessment will potentially receive higher levels of scrutiny, as their importance to society increases. We define subsurface as the earth's material including rocks, gases and fluids not exposed at the surface. Further examples of areas of geoscience addressing reproducibility include standardisation of pre-processing using open-source software in seismology (e.g., Beyreuther et al. 2010), large parts of computational geoscience (e.g., Konkol et al. 2018), or reviews in quantitative areas of geomorphology (e.g. Paola et al. 2009; Church et al. 2020).

In 2015, researchers from a number of US academic and governmental institutions undertook a workshop focused on the theme of "Geoscience Paper of the Future" identifying: (i) welldocumented datasets hosted on public repositories, (ii) documentation of software/code, including pre-processing of data and visualization steps and metadata, and (iii) documentation and availability of computational provenance for each figure/result, would significantly aid efforts to improve reproducibility (see David et al. 2016). Yet, for the most part, subsurface geoscience has lacked any formal discussions or initiatives surrounding reproducibility. Part of this absence may be related to an emphasis on direct repeated observations, which often builds on previous work (which itself has not been reproduced, creating a mixture of quantitative and qualitative data, and theoretical models (e.g., fault displacement models). These observations and models are integrated into broader studies, which include numerous case examples, and may be thought of, 
informally, as reproducibility studies combined to give a consensus. A classic example of the problem with this consensus method is sequence stratigraphic models. The model was heavily influenced from the use of 2D seismic-reflection data from the Neogene-Quaternary Mississippi fan (cf. Vail et al. 1977; Posamentier et al. 1988; Van Wagoner et al. 1990), with the central tenet being that the stratigraphic organisation of marine sedimentary sequences was or is controlled by the interaction of tectonics (subsidence and uplift) and sea-level change. Testing of and modification to this model soon ensued (e.g., Hunt \& Tucker 1992), and alternative models/interpretations (Helland-Hansen \& Gjelbeg 1994) arose. Madof et al. (2019) revisited one of the type locations (the Mississippi Fan) to re-evaluate the original models, concluding that sequence stratigraphy presents an oversimplification and called into question the concepts of accommodation-driven reciprocal sedimentation. This is not to say that the ideas within sequence stratigraphy are not useful, in fact they have been extremely important for hydrocarbon exploration. In a sense they are similar to economic models, they are sufficient as a generalisation, which provides a loose framework to study the subsurface, but have a large degree of uncertainty.

Reproducibility is an essential element of scientific work. It enables researchers to re-run and reuse experiments reported by others, learning from their successes and failures, and by doing so producing overall 'better' science. Reproducibility is vital to ensure complete reporting of all relevant aspects of scientific design, measurements, data, and analysis (see Goodman et al., 2016). Geosciences has a significant contribution to make in implementing the United Nations (UN) Sustainable Development Goals (SDGs) through collaborating with social scientists, partnering with civil society and end users, and communicating existing research to policymakers (Scown, 2020). Subsurface geoscience is core to this, where the work has a significant role in informing and supporting the delivery of projects such as CCS, nuclear waste disposal, mining for base metals and rare earth elements, and also forecasting relating to the mitigation of natural hazards (e.g., volcanic eruptions and earthquakes). In particular, those activities, which involve geoengineering, are often seen as controversial in their infancy in terms deployment or understanding (e.g., Shepherd 2009), and capital-intensive requiring oversight from governmental and intergovernmental bodies. Improved reproducibility and transparency will allow the weight of evidence presented to be evaluated more efficiently and reliably, allowing the design of a higher proportion of future studies to address actual knowledge gaps or to effectively strengthen cumulative evidence (Scown, 2020). Reproducibility and its modus operandi of transparency are critical to subsurface geoscience and should underpin the decision making for sustainable development and science communication. Transparency acts as a quality control mechanism which incentivises authors to publish results that are more robust and allows others to reproduce analyses. The aim of this study is to understand the current state of play of reproducibility in subsurface geoscience, collating geoscientists experience globally across academia, industry, and government.

\section{Methods and Data}

We use results collected from a 2020 survey of 347 geoscientists to collect views on the current state of reproducibility within subsurface geoscience. Full details of the specific questions, ethical 
considerations, and an anonymised version of the results are provided in the supplementary material of this article.

The survey utilised opportunity sampling and was publicised through the author's networks, social networks (e.g., Twitter), and geoscience-related mailing lists and conferences. Participants responded to 43 questions on topics including general questions on participants background, experiences and views on reproducibility, and view on the practicalities of reproducibility and any suggestions for improvements in subsurface geoscience going forward. All participants responded to all questions within the survey. Surveys were coded and analysed utilising a thematic approach. Findings presented provide qualitative analyses of results utilising prevalence estimates and reporting prominent themes relating to repeatability, replicability and reproducibility. The respondents are from a variety of backgrounds, including academia, governmental institutions, and multiple industries spanning mining \& quarrying, engineering geology, oil and gas, renewable energy, hydrogeology, environmental monitoring, natural hazards, and CCS.

The highest education level achieved by the participants ranged from undergraduate $(7.2 \%)$, masters $(28 \%)$, doctoral $(30.6 \%)$, to post-doctoral $(34.1 \%)$, and ranged in experience of working with subsurface data from 0 to $20+$ years. Most respondents identified as Geologists (41\%), Geophysicists (24.6\%), or a combination of the two (30.3\%). Over $85 \%$ of respondents said they publish formal literature.

\section{Results}

We found that the majority $(89.3 \%)$ of respondents broadly identified the need for improved reproducibility in geoscience, with $38.3 \%$ stating there is a minor problem, and $51 \%$ stating there is a major problem (Fig. 1). Broadly the responses were consistent across those who identified as Geologists, Geophysicists, or integrated G\&G. Though when asked a follow up question about how much of the geoscience literature is reproducible, we gathered unclear results with an approximately normal distribution of responses from $10-100 \%$, with most respondents recording between $20-80 \%$ of the literature being reproducible (Fig. 1). This suggests that although the community at large is aware there is a need to improve reproducibility, we are unsure as to how much of the literature is reproducible. Most noticeably those who write software code regularly seemed slightly more concerned/aware about the problems of reproducibility (Fig. 1).

Most respondents had tried to reproduce their own work at a later date, and $61 \%$ stated they had tried to reproduce others' published work. However, those that tried to reproduce both their own results and others results dropped to less than half. Reasons for reproducing an author's own work included: carrying-out general quality control, testing different equipment, testing different methods and conceptual models, checking different software and code, and repeating analysis after the collection of additional data. However, only $12.5 \%$ of respondents have published or attempted to publish positive or negative replication findings, with many describing difficulties relating to data access, poorly described or incomplete methodology, an inability to access proprietary software, and inaccessible and/or poor data management (see below). Behind these 
more practical issues, the survey revealed that people felt a lack of interest, hostility, and professional and/or personal cost to undertaking reproducibility studies, in particular for critiques of well-respected "seminal" papers. As one respondent noted "you are essentially critiquing work from a member of a very small close-knit community". In addition, those who have tried to publish reproducibility studies reported having been rejected at the peer-review stage for reasons including editors and reviewers not considering replication studies as novel or ground-breaking science, not providing enough data from the original study, and conflicting interests with reviewers often having connections to the original study. To better understand the barriers to improving reproducibility, we asked the participants what specific issues make published results difficult to reproduce. The most encountered difficulties included access to data (97\%), undocumented methods (92\%), confidentiality/copyright issues (85\%), along with access to code (81\%) or software (80\%) (Fig. 2). When asked what factors contribute to irreproducibility respondents identified several approximately equal issues including: reporting bias, poor methodology and analysis, questionable statistical validity, pressure to publish, poor project/experimental design, and insufficient mentoring and supervision of early-career scientists. Thankfully, fraud was viewed as the least likely factor to contribute to irreproducible publications. In particular three recurring issues came up in the comments: (i) a feeling that thorough peer-review is difficult to achieve as the reviewer typically only receives a mixture of finished figures and tables rather than the raw data in a usable and thus reproducible form, (iii) a pressure on authors to produce a larger quantity of research in less time, with the long-held mantras of "publish or perish" (e.g., Angell 1986) and "quantity over quality" (e.g., Michalska-Smith \& Allesina 2017) still felt by many, and (iii) the time allotted to peer-review has been reduced. These are known problems throughout many research fields, and it seems from this survey that these issues are also prevalent throughout geoscience.

\section{Discussion \& Conclusions}

Our results are in line with other review of reproducibility (e.g., Baker 2016), and indicate that reproducibility is a significant issue in subsurface geoscience. Below we discuss some of the corrective measures that may aid in improving reproducibility, and challenges that persist.

\section{Corrective measures \& challenges}

\section{Data}

Access to data was the most encountered difficulty identified by respondents (Fig. 2). Within the free-text comments, many respondents detailed difficulties with reviewing papers, understanding and assessing results, and comparing studies. This is a well-known issue in subsurface geoscience, particularly when confidential industry data, for example from energy companies, is being used. Such data are not only costly to acquire, but are also commercially sensitive and therefore, cannot be openly shared. One area where using open access data has proved useful for reproducibility is when testing machine learning algorithms on seismic-reflection surveys. Here use of open access data such as the popular Dutch F3 3D seismic-reflection survey (e.g., Waldeland \& Solberg 2017; Mosser et al. 2019) has allowed researchers to make direct comparisons. However, this approach is not suitable for every subfield of geoscience where a 
variety of different examples/and data locations are required and therefore, different initiatives are needed to begin to bring some standardisation across different datasets and localities.

The solution to the data access problem is in some ways simple, along with the publication itself, well-documented data sets on easy to access public repositories should be encouraged wherever possible. These shared data should also be granted Digital Object Identifiers (DOIs) so that they are index, discovered, and so credit it given to those compiling them. However, there are difficulties and complexities in trying to rectify this issue. Do we only publish work where data is open-access, or do we accept some studies using commercially sensitive data are useful to geoscience even if we cannot reproduce them? Some of the best data, in localities most academics could never fund themselves, belongs to private enterprise. We could, therefore, make critical, possibly even live-saving observations and have and continue to garner knowledge from them. However, what's the value if there is no real clarity as to the robustness of the analysis, or if the results are not reproducible due to confidentiality in the methods employed? Where do we draw the line? This is a question for the community, but one potential solution we would suggest would be to have some form of traffic light system on publications so readers can easily access if the data is open or not.

\section{Methods, conceptual bias, and a framework for minimum publishing standards}

Separation of interpretations from raw data and/or observation, access to code, conceptual uncertainty, along with lack of detailed methodology framework repeatedly came up as major issues in the survey (Fig. 2). Subsurface geosciences benefit from a breadth of geological, geophysical, biological, and chemical data collected by both industry and academia. As a result of this the methods, software and code used in the analysis of data span many different disciplines. Like Bond et al. (2007) our survey highlights the importance of conceptual bias/uncertainty and the impact this may be having on reproducibility. This highlights three important points: (i) uncertainty should be quantitatively or qualitatively captured in subsurface geoscience studies, where data quality and or limitations in current knowledge mean there is no correct conclusion (e.g., Alcalde et al. 2017), and (ii) methods should be encouraged to standardise measurements (where possible) so studies can be compared easily (e.g., Clare et al. 2019), and (iii) methods should promote the documenting of alternate scenarios (e.g., Bentley \& Smith 2008). Clare et al. (2019) demonstrates how a consistent global approach to the measurement of subaqueous landslides enables comparison of research across scales and geological environments and has been adopted by much of the research community and governmental organisations. Bentley \& Smith (2008) showcase how modelling subsurface reservoirs using multiple deterministic realisations, rather than anchoring on one interpretation, increases the chance of capturing the full uncertainty range within a hydrocarbon or CCS project.

These results suggest a set of minimum standards for publications focusing on detailed documentation of methods and conceptual uncertainty would be beneficial to enhancing reproducibility efforts. This does not necessarily mean lengthening the paper, but instead 
providing adequate and detailed supplementary material for readers who are aiming to reproduce work. We therefore draw similar conclusions to the "Geoscience Paper of the Future" (see David et al. 2016), calling for further transparency of methods employed. Ironically many publishers have strict standards in place for type setting and formatting of manuscripts, while often neglecting the more important aspects of the science. Hence, we would echo the thoughts of David et al. (2016) on the matter of implementing a minimum standards framework for published work.

\section{Incentives}

Currently, publishing a paper in a peer-reviewed journal is considered the main output of a research project. This is because journal publication underpins citations metrics such as the $\mathrm{h}$ index, which are commonly used to assessed research outputs and researchers (e.g., College \& James 2015). The more papers a researcher publishes and the more these papers are cited, the greater the esteem and the higher the likelihood they will be hired, promoted, or rewarded. As such, from a metrics standpoint it is more important to publish cited papers, regardless of the quality and, certainly, the reproducibility of the work. Another citation metric is the Journal Impact Factor or 'JIF', which essentially attempts to communicate the quality of a journal and thus, the quality of the papers published within that journal. Many general (e.g., Nature, Science) or fieldspecific (e.g., GEOLOGY, Nature Geoscience), high-JIF journals are highly prized by scientists for the reasons outlined above; however, the conflict here is that these journals typically favour papers containing 'novel' or 'ground-breaking' work of general interest, with studies focused on reproducibility or replication less likely to be published. There is also less value attached to publishing so-called negative results, despite the underpinning studies being central to refining and improving the scientific method and assessing the robustness (and in some cases safety) of already published work.

The knowledge, adoption, and quality of reproducibility in subsurface geoscience could thus be increased by modifying current incentive frameworks in academic and non-academic workplaces. Greater emphasis and value needs placing on research methods and transparency, and the overall robustness of results, as at least partly defined by how reproducible they are. This could be done by incentivising open and transparent science, the basis for reproducibility, in hiring, promotion, and general assessment frameworks. For example, credit (in whichever form is appropriate) should be given for committing to providing datasets and derivative data (e.g., seismic interpretation horizons), code, and software, for example, publicly available. This can be achieved by placing these data in public repositories that issue DOls.

\section{Reflections}

Today there exists the digital infrastructure to support the publishing and archiving of results and data to enable geoscientists to carry out and assess reproducibility. There will always be hurdles to overcome however, we believe it is important for the geoscience community to embrace the importance of reproducibility, particularly as our science will likely be put under the spotlight in the coming decades with increased activities in areas such as CCS, nuclear waste disposal, and 
natural hazard prediction. We hope the survey results provide an initial catalyst for conversations within research, industry, and government, around how we can begin to improve working practices and dissemination of geoscience to specialists, practitioners, policy makers, and the public. Our results also highlight the notion of reproducibility and the requirement for substantive and trustworthy data beyond geoscience to broader the disciplines of natural and social sciences.

\section{A Whole-System Approach to Improving Reproducibility}

Scientific research is a human endeavour, and therefore fallible, subject to cognitive biases brought by scientists themselves to their work, and shaped by incentives that influence how scientists behave (Munafò et al. 2020). Understanding the nature of the research ecosystem, and the role played by individual researchers, institutions, funders, publishers, learned societies and other sectoral organisations is key to improving the quality and robustness of the research we produce (Munafò et al. 2017). Coordinating the efforts of all of these agents will be necessary to ensure incentives are aligned and promote appropriate behaviours that will drive research quality, and reward the varied contributions that diverse individuals make to modern scientific research.

The UK Reproducibility Network (UKRN; www.ukrn.org) was established to support the coordination of these agents. It comprises local networks of researchers, institutions that have formally joined the network, and external stakeholders comprising funders, publishers, learned societies and a range of other organisations. It works to coordinate activity within and between these groups, with the overarching goal of identifying and implementing approaches that serve to improve research quality. Rather than treating issues such as the low reproducibility of published research findings as a static problem to be solved, it recognises the dynamic and evolving nature of research practices (and research culture more generally), and in turn the need to adopt a model of continual improvement.

One area of current focus is open research - and transparency in research more broadly - recognised by the UK government R\&D People and Culture Strategy as "integral to a healthy research culture and environment". This allows the recognition of more granular, intermediate research outputs (e.g., data, code) from diverse contributors to the research process. However, it has also been argued to serve as a quality control process, allowing external scrutiny of those outputs, and in turn creating an incentive to ensure robust internal quality assurance processes (Munafò et al., 2014). However, fully embedding open research practices will require infrastructure and training, incentives (for example, recognition in hiring and promotion practices), support from funders and publishers, and more.

We also need to recognise that well-intentioned changes to working practices may not work as intended or, worse still, may have unintended consequences. For example, they may serve to exacerbate existing inequities if only well-resourced research groups are able to engage with them fully, particularly if this engagement becomes a factor that influences grant and publishing success. We therefore need to evaluate the likely impact of changes to working practices on under-represented and minoritised groups, and continue to evaluate the impact of these changes once implement. Again, we need to move to a model of continual improvement which links innovation to evaluation. Meta-research - also known as research on research - is now an established area of inquiry that allows us to use scientific 


\section{References}

Alcalde, J., Bond, C.E., Johnson, G., Ellis, J.F. and Butler, R.W., 2017. Impact of seismic image quality on fault interpretation uncertainty. GSA Today.

Angell, M., 1986. Publish or perish: a proposal. Annals of Internal Medicine, 104(2), pp.261-262.

Baker, M., 2016. Reproducibility crisis. Nature, 533(26), pp.353-66.

Bentley, M. and Smith, S., 2008. Scenario-based reservoir modelling: the need for more determinism and less anchoring. Geological Society, London, Special Publications, 309(1), pp.145-159.

Beyreuther, M., Barsch, R., Krischer, L., Megies, T., Behr, Y. and Wassermann, J., 2010. ObsPy: A Python toolbox for seismology. Seismological Research Letters, 81(3), pp.530-533.

Bond, C.E., Gibbs, A.D., Shipton, Z.K. and Jones, S., 2007. What do you think this is?"Conceptual uncertainty"in geoscience interpretation. GSA today, 17(11), p.4.

Camerer, C.F., Dreber, A., Forsell, E., Ho, T.H., Huber, J., Johannesson, M., Kirchler, M., Almenberg, J., Altmejd, A., Chan, T. and Heikensten, E., 2016. Evaluating replicability of laboratory experiments in economics. Science, 351(6280), pp.1433-1436.

Church, M., Dudill, A., Venditti, J.G. and Frey, P., 2020. Are results in geomorphology reproducible?. Journal of Geophysical Research: Earth Surface, 125(8), p.e2020JF005553.

Clare, M., Chaytor, J., Dabson, O., Gamboa, D., Georgiopoulou, A., Eady, H., Hunt, J., Jackson, C., Katz, O., Krastel, S. and León, R., 2019. A consistent global approach for the morphometric characterization of subaqueous landslides. Geological Society, London, Special Publications, 477(1), pp.455-477.

David, C.H., Gil, Y., Duffy, C.J., Peckham, S.D. and Venayagamoorthy, S.K., 2016. An introduction to the special issue on Geoscience Papers of the Future. Earth and Space Science, 3(10), pp.441-444.

Goodman, S.N., Fanelli, D. and loannidis, J.P., 2016. What does research reproducibility mean?. Science translational medicine, 8(341), pp.341ps12-341ps12.

Helland-Hansen, W. and Gjelberg, J.G., 1994. Conceptual basis and variability in sequence stratigraphy: a different perspective. Sedimentary Geology, 92(1-2), pp.31-52.

Hunt, D. and Tucker, M.E., 1992. Stranded parasequences and the forced regressive wedge systems tract: deposition during base-level'fall. Sedimentary Geology, 81(1-2), pp.1-9.

Konkol, M., Kray, C. and Pfeiffer, M., 2019. Computational reproducibility in geoscientific papers: Insights from a series of studies with geoscientists and a reproduction study. International Journal of Geographical Information Science, 33(2), pp.408-429.

Madof, A.S., Harris, A.D., Baumgardner, S.E., Sadler, P.M., Laugier, F.J. and Christie-Blick, N., 2019. Stratigraphic aliasing and the transient nature of deep-water depositional sequences: Revisiting the Mississippi Fan. Geology, 47(6), pp.545-549.

Michalska-Smith, M.J. and Allesina, S., 2017. And, not or: quality, quantity in scientific publishing. PloS one, 12(6), p.e0178074.

Milne, G.A., Gehrels, W.R., Hughes, C.W. and Tamisiea, M.E., 2009. Identifying the causes of sea-level change. Nature Geoscience, 2(7), pp.471-478. 
Mosser, L., Oliveira, R. and Steventon, M., 2019, June. Probabilistic seismic interpretation using Bayesian neural networks. In 81st EAGE Conference and Exhibition 2019 (Vol. 2019, No. 1, pp. 1-5). European Association of Geoscientists \& Engineers.

Munafò, M., Noble, S., Browne, W.J., Brunner, D., Button, K., Ferreira, J., Holmans, P., Langbehn, D., Lewis, G., Lindquist, M. and Tilling, K., 2014. Scientific rigor and the art of motorcycle

maintenance. Nature biotechnology, 32(9), pp.871-873.

Munafò, M.R., Chambers, C.D., Collins, A.M., Fortunato, L. and Macleod, M.R., 2020. Research culture and reproducibility. Trends in Cognitive Sciences, 24(2), pp.91-93.

Munafò, M.R., Nosek, B.A., Bishop, D.V., Button, K.S., Chambers, C.D., Du Sert, N.P., Simonsohn, U., Wagenmakers, E.J., Ware, J.J. and loannidis, J.P., 2017. A manifesto for reproducible science. Nature human behaviour, 1(1), pp.1-9.

Nosek, B.A. and Errington, T.M., 2017. Reproducibility in cancer biology: Making sense of replications. Elife, 6, p.e23383.

Open, S.C., 2015. Psychology. Estimating the reproducibility of psychological science. Science, 349(6251), p.aac4716.

Paola, C., Straub, K., Mohrig, D. and Reinhardt, L., 2009. The "unreasonable effectiveness" of stratigraphic and geomorphic experiments. Earth-Science Reviews, 97(1-4), pp.1-43.

Posamentier, H.W. and Vail, P.R., 1988. Eustatic controls on clastic deposition II-sequence and systems tract models.

Scown, M.W., 2020. The Sustainable Development Goals need geoscience. Nature Geoscience, 13(11), pp.714-715.

Shepherd, J.G., 2009. Geoengineering the climate: science, governance and uncertainty. Royal Society.

Vail, P.R., Mitchum Jr, R.M. and Thompson III, S., 1977. Seismic stratigraphy and global changes of sea level: Part 4. Global cycles of relative changes of sea level.: Section 2. Application of seismic reflection configuration to stratigraphic interpretation.

Van Wagoner, J.C., Mitchum, R.M., Campion, K.M. and Rahmanian, V.D., 1990. Siliciclastic sequence stratigraphy in well logs, cores, and outcrops: concepts for high-resolution correlation of time and facies.

Waldeland, A.U. and Solberg, A.H.S.S., 2017, June. Salt classification using deep learning. In 79th eage conference and exhibition 2017 (Vol. 2017, No. 1, pp. 1-5). European Association of Geoscientists \& Engineers. 
Do you think there is a reproducibility problem in subsurface geoscience?

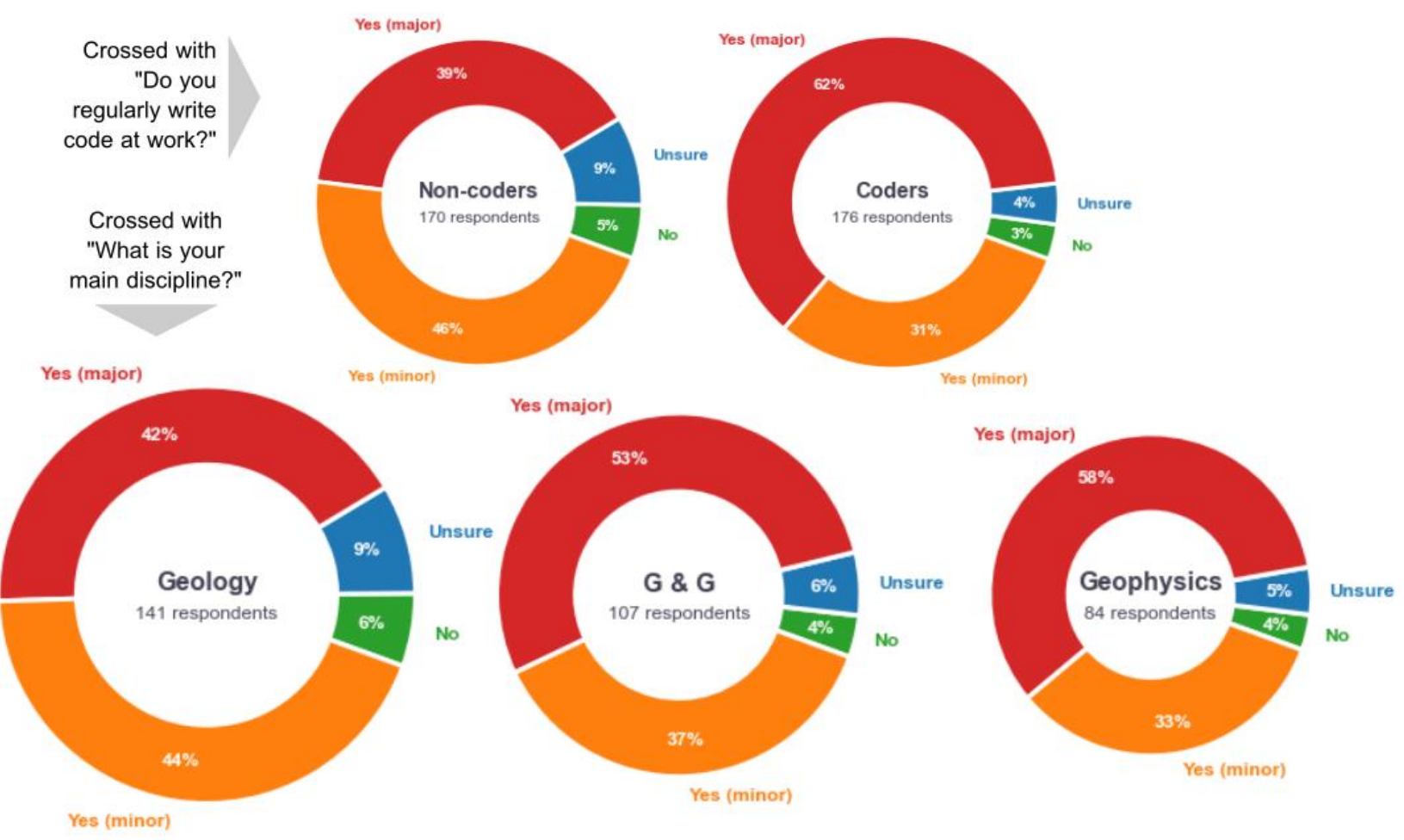

How much of the geoscience literature is reproducible?

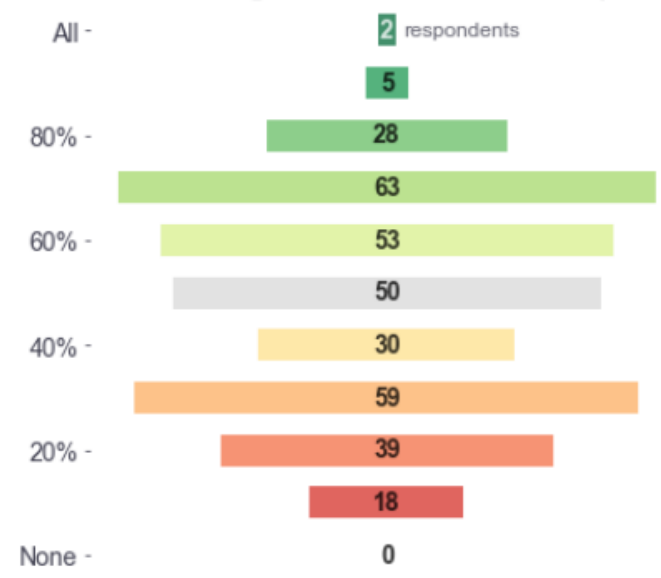

Have you tried to reproduce...

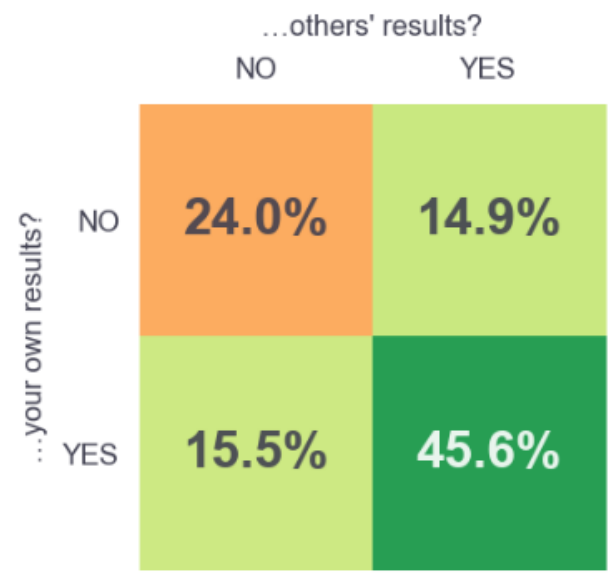

Figure 1: Overview of survey results. G\&G pertains to survey participants that identify as both Geologists and Geophysicists. Coders refers to those in the sample that said they coded regularly. 
What practicalities make published results difficult to reproduce?

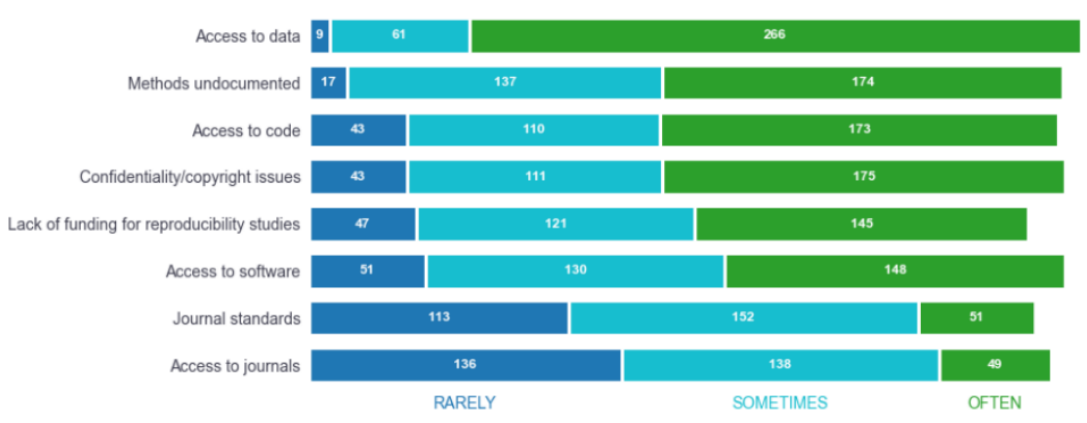

What factors contribute to irreproducible publications?

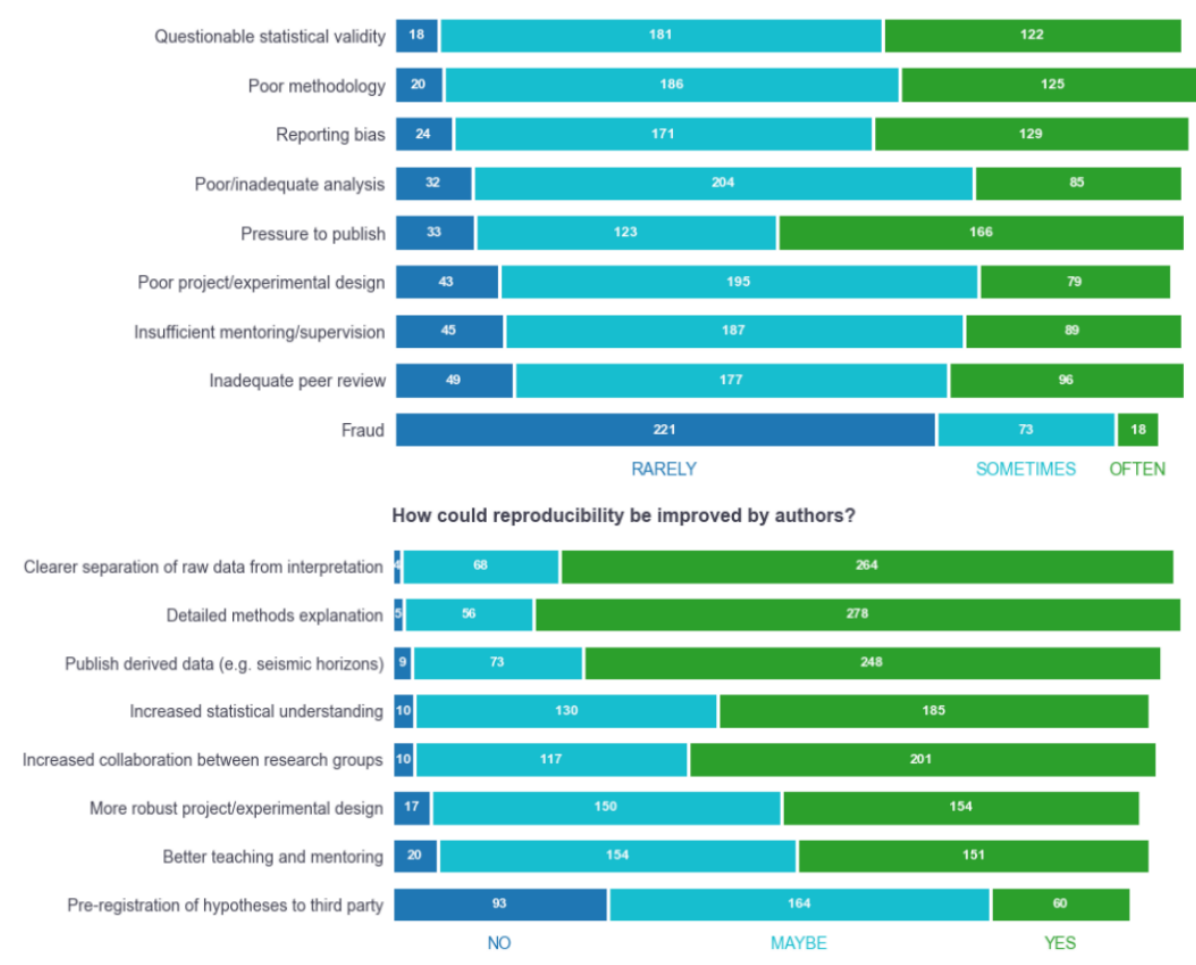

Figure 2: Overview of survey results (II) 


\section{Funding}

This work was undertaken by the first author as part of post-graduate studies at Imperial College London. The work was carried-out on a voluntary basis by all authors.

Data availability

An anonymised version of the results can be found in the below supplementary material. 